\title{
Therapeutic targeting of SPINK1-positive prostate cancer
}

\author{
Bushra Ateeq ${ }^{1,2}$, Scott A. Tomlins ${ }^{1,2}$, Bharathi Laxman ${ }^{1,2}$, Irfan A. Asangani ${ }^{1,2}$, Qi Cao ${ }^{1,2}$, \\ Xuhong Cao ${ }^{1,5}$, Yong $\mathrm{Li}^{1,2}$, Felix Y. Feng ${ }^{1,3,7}$, Kenneth J. Pienta ${ }^{1,4,7}$, Sooryanarayana \\ Varambally $1,2,6$, and Arul M. Chinnaiyan $1,2,5,6,7, \#$ \\ ${ }^{1}$ Michigan Center for Translational Pathology, Ann Arbor, MI, 48109, USA \\ ${ }^{2}$ Department of Pathology, University of Michigan, Ann Arbor, MI, 48109, USA \\ ${ }^{3}$ Department of Radiation Oncology, University of Michigan, Ann Arbor, MI, 48109, USA \\ ${ }^{4}$ Department of Medicine, University of Michigan, Ann Arbor, MI 48109 \\ ${ }^{5}$ Howard Hughes Medical Institute, University of Michigan Medical School, Ann Arbor, MI, 48109, \\ USA \\ ${ }^{6}$ Department of Urology, University of Michigan, Ann Arbor, MI, 48109, USA \\ ${ }^{7}$ Comprehensive Cancer Center, University of Michigan Medical School, Ann Arbor, MI, 48109, \\ USA
}

\section{Abstract}

The discovery of recurrent gene fusions involving Erythroblastosis virus E26 transformationspecific (ETS) family transcription factors in approximately $50 \%$ of prostate cancers provides a basis for the molecular subclassification of prostate cancer. Previously, we showed that marked over-expression of SPINKI (serine peptidase inhibitor, Kazal type 1), which encodes a secreted serine protease inhibitor, defines an aggressive molecular subtype of ETS fusion-negative prostate cancers (SPINK1 ${ }^{+} /$ETS $^{-}, \sim 10 \%$ of all prostate cancers). Here, we examined the potential of SPINK1 as an extracellular therapeutic target in prostate cancer. We demonstrate that recombinant SPINK1 protein (rSPINK1) stimulates cell proliferation in benign RWPE and cancerous prostate cells. RWPE cells treated with rSPINK1 or conditioned medium from 22RV1 prostate cancer cells $\left(S P I N K 1^{+} / E T S^{-}\right)$showed significantly increased cell invasion and intravasation. Knockdown of SPINK1 in 22RV1 cells inhibited cell proliferation, cell invasion, and tumor growth in xenograft assays. Importantly, 22RV1 cell proliferation, invasion and intravasation were attenuated by an anti-SPINK1 monoclonal antibody $(\mathrm{mAb})$. We also demonstrate that SPINK1 partially mediates its neoplastic effects through interaction with the epidermal growth factor receptor (EGFR). Administration of anti-SPINK1 mAb or anti-EGFR mAb (cetuximab) to mice bearing 22RV1 xenografts attenuated tumor growth by over $60 \%$ and $40 \%$ alone, respectively, and approximately $75 \%$ when combined, without affecting PC3 xenograft (SPINK1-/ETS $)$ growth. Taken together, this study qualifies SPINK1 as a therapeutic target in a subset of patients with $S P I N K 1^{+} /$ETS $^{-}$ prostate cancer. Similar to antibody targeting of ERBB2 in a subset of breast cancers, our results

\footnotetext{
${ }^{\#}$ To whom correspondence should be addressed: Arul M. Chinnaiyan, M.D., Ph.D. Investigator, Howard Hughes Medical Institute Department of Pathology and Urology University of Michigan Medical School 1400 E. Medical Center Drive, 5316 UMCCC Ann Arbor, MI-48109 Phone: 734-615-4062 Fax: 734-615-4498 arul@umich.edu.

Author Contribution

B.A., S.A.T, and A.M.C. designed the research plan and wrote the manuscript; B.A., B.L., Q.C., and X.C., performed the in vitro experiments; I.A.A. performed CAM assays; B.A. performed in vivo xenograft experiments; B.A., B.L., I.A.A., S.A.T., F.Y.F., K.J.P. S.V. and A.M.C. analyzed data.

Competing Interests

The University of Michigan has filed for patents on the SPINK1, on which A.M.C., and S.A.T. are named as inventors. The diagnostic field of use has been licensed to Gen-Probe, Inc. Gen-Probe was not involved in the design or funding of these studies.
} 
provide rationale for both the development of humanized anti-SPINK1 monoclonal antibodies and evaluation of EGFR inhibition in $S P I N K 1^{+} / E T S^{-}$prostate cancers.

\section{INTRODUCTION}

Therapies targeted against specific molecular alterations present only in cancer cells have revolutionized the treatment of several cancers. For example, targeting ERBB2, which is amplified in approximately $20 \%$ of breast cancers, with the humanized monoclonal antibody $(\mathrm{mAb})$ trastuzumab (Herceptin) has resulted in improved survival for breast cancer patients. Although organ confined prostate cancer is highly curable, more than 32,000 U.S. men are expected to die of metastatic prostate cancer in 2010 (1). While multiple approved therapies (and newer agents in late stage development) target the androgen signaling axis in metastatic disease, additional targeted therapies are lacking.

We previously employed a bioinformatics approach termed Cancer Outlier Profile Analysis (COPA) to systematically prioritize genes with marked over-expression in a subset of cancers (outlier expression), which identified outlier expression of the ETS family members $E R G$ and ETVI in a subset of prostate cancers across multiple gene expression profiling studies. This strategy led to the discovery of recurrent gene fusions involving the 5 ' untranslated region of the androgen regulated gene TMPRSS2 with ETS transcription factors (ERG, ETV1, ETV4 or ETV5) (2-5). Subsequent studies in vitro and in vivo have demonstrated a driving role for ETS fusions in prostate oncogenesis and cancer progression (6-9).

Subsequently, we employed a "meta-outlier approach", which used COPA to prioritize genes that consistently showed high ranking outlier expression across multiple profiling studies, and identified SPINKI, as a high ranking meta-outlier in prostate cancer that also showed mutually exclusive outlier expression with $E R G$ and $E T V 1$ across prostate cancer profiling multiple studies (10). SPINK1, also known as pancreatic secretory trypsin inhibitor (PSTI) or tumor-associated trypsin inhibitor (TATI), encodes a 56-amino acid peptide thought to protect the pancreas from auto-digestion by preventing premature activation of pancreatic proteases (11). Apart from its normal expression in pancreatic acinar cells, SPINK1 mRNA has been reported to be expressed in various human cancers (12-18), and increased serum SPINK1 concentration has been correlated with poor prognosis in some studies $(12,13,17)$. The prostate gland also secretes a variety of serine proteases, most notably the kallikrein enzyme PSA, but also trypsin (19). Thus, SPINK1 may have a role in modulating the activity of cancer-related proteases in other tissues besides the pancreas.

Previously, we confirmed the mutually exclusive over-expression of SPINK1 and ETS gene fusions using a combined immunohistochemistry (for SPINK1) and FISH approach (for ETS fusions) across multiple independent cohorts, and demonstrated that SPINK1 outlierexpression is associated with an aggressive subset of prostate cancers (10). We also demonstrated that SPINKI outlier expression can be detected non-invasively in urine and contributes to a multiplexed panel of biomarkers, which outperforms serum PSA for prostate cancer diagnosis in patients presenting for needle biopsy $(10,20)$. Our combined analyses of over 1,500 prostate cancer cases demonstrated SPINK1 outlier-expression in approximately $10 \%$ of all PSA-screened prostate cancers, which were invariably negative for ETS gene fusions $\left(S P I N K 1^{+} / E T S^{-}\right)(10)$. Furthermore, SPINKI ${ }^{+}$tumors show shorter PSA recurrence free survival in prostatectomy-treated patients $(10)$ and shorter progression free survival in endocrine-treated patients (21).

Unlike ETS gene fusions that lead to the over-expression of a transcription factor (which are difficult to target therapeutically), SPINKI encodes an extracellular secreted protein, and 
thus is potentially more amenable to therapeutic targeting. Here we qualify SPINK1 as a therapeutic target in $S P I N K 1^{+} / E T S^{-}$prostate cancer, and demonstrate the therapeutic potential of an anti-SPINK1 monoclonal antibody in pre-clinical models. Additionally, we demonstrate that SPINK1 mediates its oncogenic effects in part through EGFR, and an antiEGFR monoclonal antibody shows in vitro and in vivo activity in $S P I N K 1^{+}$prostate cancer.

\section{RESULTS}

\section{SPINK1 as an autocrine factor in prostate cancer}

To further investigate the role of SPINK1 in prostate cancer, we determined the effects of exogenous SPINK1 on invasion and proliferation using recombinant 6XHis-tagged SPINK1 protein (rSPINK1) (Fig. S1-A) or conditioned media (CM) collected from 22RV1 prostate cancer cells $\left(\right.$ SPINK1 $\left.{ }^{+} / E T S^{-}\right)$(Fig. S1-B) (10). We treated benign immortalized RWPE prostate epithelial cells and DU145 and PC3 prostate cancer cells (both of which are SPINK1 $\left.1^{-} / E^{-} S^{-}\right)$with rSPINK1 $(10 \mathrm{ng} / \mathrm{ml})$, which resulted in a significant increase in cell proliferation (Fig. 1A). We next characterized the effect of rSPINK1 or 22RV1 CM on cell invasion using a Boyden chamber Matrigel invasion assay. As shown in Figure 1B, addition of rSPINK1 or 22RV1 CM to RWPE cells significantly increased invasion $(P=0.003$ and 0.0009 , respectively). Similar effects were observed when MCF7 breast cancer cells were treated with rSPINK1 or 22RV1 CM (Fig. S1-C). Multiple recombinant 6XHis tagged control proteins or CM collected from RWPE or LNCaP prostate cancer cells did not induce invasion in RWPE cells (Fig. S1-D, S2).

We previously showed that transient siRNA mediated knock-down of SPINK1 in 22RV1 cells decreased cell invasion (10). Here, we extended these results by demonstrating that the addition of rSPINK1 or 22RV1 CM rescued the invasive phenotype of 22RV1 cells in which SPINK1 was knocked down (Fig. 1C, $P=0.001$ for both rSPINK1 or 22RV1 CM).

We next investigated whether the exogenous effect of SPINK1 on cell proliferation and invasion is dependent on protease inhibitory activity of trypsin (which has been shown to be simultaneously expressed with SPINK1 in different tumor types $(17,22))$ or PSA. Initial experiments demonstrated that PRSSI (trypsinogen) mRNA expression in 22RV1 cells is relatively low (Fig. S3-A), although a significant increase in PRSS1 transcript was observed in siRNA mediated SPINK1 knockdown 22RV1 cells (Fig. S3-B). However, as shown in Figure S3-C, stimulation of 22RV1 cells with rSPINK1 or EGF did not affect trypsin expression. siRNA mediated knockdown of PRSS1 in 22RV1 cells also had no effect on invasion (Fig. S3-D \& E). Similarly, stimulation of 22RV1 cells with rSPINK1 or EGF did not affect PSA expression (Fig. S4-A). Finally, blocking PSA using a monoclonal antibody did not significantly inhibit 22RV1 cell invasion (Fig. S4-B). Together, these findings demonstrate that extracellular SPINK1 induces prostate cancer cell proliferation and invasion independent of protease inhibitory activity of trypsin or PSA. Although effects on other proteases cannot be excluded, our results suggest that SPINK1 is an autocrine proproliferative and pro-invasive factor with effects independent of protease activity.

\section{The role of SPINK1 in cell proliferation and invasion}

To further investigate the role of SPINK1 in cell proliferation and invasion, we generated shRNA against SPINK1 and established stable 22RV1 cells where SPINK1 was silenced ( $\operatorname{sh}$ SPINK1). Knockdown of SPINKI in both pooled and clonal shSPINKI cells compared to non-targeting control cells (shNS cells) was confirmed at the RNA level by quantitative PCR (more than $80 \%$ in both), as well as at the protein level by immunofluorescence staining using an antibody against SPINK1 (Fig. 1D). Next, we investigated the role of SPINK1 in cell invasion and motility using shSPINKI cells. As anticipated, shSPINKI cells showed 
decreased cell invasion by more than $75 \%$ in a Boyden chamber Matrigel assay compared to non-specific vector control $(\operatorname{sh} N S)$ cells $($ Fig. 1E; $P=0.002)$. Reduction of cell motility in a bead motility assays was also observed in shSPINKI cells as compared to shNS cells (Fig. 1E; top panel).

To investigate the role of SPINK1 in cell proliferation, we carried out assays using pooled shSPINK1, the clone with the greatest SPINK1 knockdown (shSPINKl clone 11), and shNS cells. Both pooled (55\% reduction) and clonal shSPINKI cells (66\% reduction) showed significantly decreased proliferation compared to sh $N S$ cells (Fig. 1F; $P=0.00002$ in both cases). Further, shSPINK1 cells showed decreased soft agar colony formation when compared to shNS cells (Fig. 1G).

\section{In vitro targeting of SPINK1 using a monoclonal antibody}

As our results above demonstrate a role for SPINK1 in invasion and proliferation, and SPINK1 is an extracellular secreted protein, we hypothesized that a monoclonal antibody $(\mathrm{mAb})$ against SPINK1 may be able to directly target $S P I N K 1^{+} / E T S^{-}$prostate cancer cells. Thus, we tested the effects of an anti-SPINK1 mAb on 22RV1 cell proliferation and invasion. Anti-SPINK1 $\mathrm{mAb}(0.5$ and $1 \mu \mathrm{g} / \mathrm{ml})$ significantly inhibited $22 \mathrm{RV} 1$ cell proliferation by 40 and $50 \%$ respectively compared to a control monoclonal IgG antibody (Fig. 2A \& B; $P=0.0001$ and $P=0.0007$ respectively). However, anti-SPINK1 antibody had no effect on DU145 and PC3 cell proliferation.

In addition to inhibiting proliferation, anti-SPINK $1 \mathrm{mAb}(0.5$ and $1 \mu \mathrm{g} / \mathrm{ml})$ significantly attenuated cell invasion by $69 \%$ and $81 \%$ respectively as compared to a control $\mathrm{IgG} \mathrm{mAb}$ in 22RV1 cells (Fig. 2C; $P=0.002$ and $P=0.007$ respectively). Similar to 22RV1, which is an androgen signaling independent derivative of primary CWR22 human prostate xenograft tumors, we also investigated CWR22Pc cells, an androgen signaling dependent derivative of CWR22 (23), which also express high levels of SPINK1. As expected CWR22Pc cell invasion was blocked by 47 and $54 \%$ by anti-SPINK $1 \mathrm{mAb}$ at 0.5 and $1 \mu \mathrm{g} / \mathrm{ml}$ of SPINK1 mAb concentration (Fig. 2C; $P=0.003$ and $P=0.002$ respectively). Importantly, the antiSPINK1 mAb had no significant effect on invasion of $S P I N K 1^{-}$prostate cancer cell lines including PC3, DU145, LNCaP or VCaP (Fig. 2C). Finally, the anti-SPINK1 mAb attenuated 22RV1 cell motility compared to IgG control, but had no effect on PC3 $\left(\right.$ SPINK1 ${ }^{-} /$ETS $\left.^{-}\right)$cell motility (Fig. S5-A).

\section{Oncogenic effects of SPINK1 in part through interaction with EGFR}

SPINK1 has a similar structure as epidermal growth factor (EGF), with approximately $50 \%$ sequence homology and three intrachain disulfide bridges $(24,25)$. To characterize potential SPINK1 and EGFR interaction, we overexpressed EGFR in human embryonic kidney cells (HEK) 293 cells and incubated the lysates with SPINK1-GST, GST or GST-VEGF Receptor 2 (GST-VEGFR) recombinant proteins. We observed a strong interaction between SPINK1GST and EGFR but not with GST alone or GST-VEGFR recombinant protein (Fig. 3A; top panel). Endogenous SPINK1 and EGFR interaction was not detected by immunoprecipitation and immunoblotting in 22RV1 cells, due to secretory nature of the SPINK1 protein. However, addition of GST-SPINK1 to 22RV1 cells followed by immunoprecipitation and immunoblotting confirmed the interaction of SPINK1 and endogenous EGFR in 22RV1 cells (Fig. 3A; bottom panel).

To further delineate the role of EGFR mediation of SPINK1 in prostate cancer, we next assessed whether exogenous SPINK1 was capable of inducing EGFR phosphorylation (similar to the cognate ligand EGF). Stimulating 22RV1 cells with rSPINK1 resulted in EGFR phosphorylation, although weaker than that observed with EGF (Fig. 3B). 
Interestingly, rSPINK1 stimulation resulted in sustained EGFR phosphorylation over a 90 minute time course, while EGF resulted in strong EGFR phosphorylation which diminished after only $10 \mathrm{~min}$. Similarly, stable shSPINK1 knockdown 22RV1 cells (pooled and clonal) showed decreased phosphorylated EGFR (pEGFR), with slightly decreased total EGFR (possibly due to EGFR degradation) (Fig. S6-A). Finally, we demonstrate that rSPINK1 is able to induce dimerization of EGFR, although more weakly than EGF (Fig. S6-B).

We next examined the functional consequences of SPINK1-EGFR interaction in the context of $S P I N K 1^{+}$prostate cancer using $22 \mathrm{RV} 1$ cells. Transient knockdown of EGFR (Fig. S5-B) blocked 22RV1 cell invasion by 75\% (Fig. 3C; $P=0.004$ ) which was partially rescued by addition of exogenous SPINK1. A similar effect of EGFR knockdown was observed in RWPE cells treated with recombinant SPINK1 (Fig. 3D; $P=0.014$ and $P=0.021$ respectively). These results suggest that some, but not all of SPINK1's effects are mediated by EGFR.

As monoclonal antibodies to EGFR are FDA approved for certain cancers, we sought to determine whether EGFR blockade could inhibit the oncogenic effects of SPINK1. We first demonstrated that $\mathrm{mAb}$ to EGFR (cetuximab, C225) blocked the cell invasive effects of rSPINK1 in RWPE cells (Fig. 3E). C225 also blocked cell invasion of $S P I N K 1^{+} 22 \mathrm{RV} 1$ cells but not in SPINK1 ${ }^{-}$cell lines DU145, PC3, LNCaP or VCaP (Fig. 3F). Combining $\mathrm{mAbs}$ to SPINK1 and EGFR had an additive effect in the inhibition of 22RV1 cell invasion (Fig. 3G; $P=0.001$ ). In contrast to mAb to SPINK1 (Fig. 2A), C225 had no effect on 22RV1 cell proliferation or PC3 and DU145 cells proliferation (Fig. 3H). Together, these experiments suggest that SPINK1 has both EGFR-dependent and EGFR-independent functions in prostate cancer.

As a preliminary exploration of the downstream signaling pathways involved in the SPINK1-EGFR axis, we studied the mitogen-activated protein kinase (MAPK) and protein kinase B/AKT pathways in stable SPINK1 knockdown 22RV1 cells (shSPINK1 clone 11). We observed decreased pMEK, pERK and pAKT in stable shSPINKI cells compared to control shNS cells (Fig. S5-C). Likewise, 22RV1 cells treated with SPINK1 mAb antibody showed decreased pERK (Fig. S5-D). These observations provide the foundation for further studies of the SPINK1-EGFR axis.

\section{The role of SPINK1 in vivo and as a therapeutic target}

Our in vitro studies demonstrated that SPINK1 mediates cell proliferation and invasion in $S P I N K 1^{+}$prostate cancer cells, and suggested that a monoclonal antibody can target extracellular SPINK1. To investigate the role of SPINK1 in intravasation, a key step involved in the process of metastasis, we employed a chick chorioallantoic membrane (CAM) model system (26) and demonstrate that rSPINK1 induced intravasation of benign RWPE cells (Fig. 4A). Similarly, SPINK1 mAb and C225 significantly inhibit 22RV1 cell intravasation $(P=0.01$ and $P=0.03$ respectively), but did not significantly inhibit PC 3 cell intravasation (Fig. 4B \& C).

To qualify SPINK1 as a potential therapeutic target in vivo, we implanted pooled shSPINK1luciferase and shNS-luciferase (luc) 22RV1 cells in nude male mice. Importantly, at both 4 and 5 weeks post-implantation, 22RV1-shSPINKI-luc cells formed significantly smaller tumors (55\% reduction at week $4, P=0.008$; and $63 \%$ reduction at week $5, P=0.013$ ) compared to shNS-luc cells (Fig. 4D \& 4H).

To demonstrate preclinical efficacy of the anti-SPINK1 $\mathrm{mAb}$, nude mice implanted with 22RV1-luciferase cells were treated with either anti-SPINK1 mAb, or an isotype matched monoclonal IgG (10 mg/kg body weight) twice a week. As shown in Figure 4E \& I, 
administration of anti-SPINK1 mAb mono-therapy resulted in a $61 \%$ reduction of tumor burden at week $4(P=0.015)$ and $58 \%$ reduction at week $5(P=0.015)$. As SPINK1 mediates its oncogenic effects in part through EGFR, we similarly assessed the mAb to EGFR (C225) using the same dosage schedule. $\mathrm{C} 225$ treatment resulted in a $41 \%$ reduction at week 4 $(P=0.04)$ and $37 \%$ reduction at week $5(P=0.02)($ Fig $4 \mathbf{E} \& \mathbf{I})$. By combining mAbs to SPINK1 and EGFR, we observed an additive effect in vivo showing a $74 \%$ and $73 \%$ reduction in the growth of 22RV1 xenografts at week $4(P=0.01)$ and $5(P=0.003)$

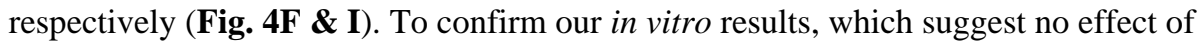
SPINK1 or EGFR inhibition on SPINKI ${ }^{-}$prostate cancer, we performed a similar xenograft study using PC3 cells. As expected, neither SPINK1 mAb nor C225 significantly inhibited tumor growth in PC3 xenografted mice (Fig. 4G \& 4I).

A significant decrease in Ki-67 positive immunostained nuclei was also observed in the SPINK1 mAb treated group as compared to the control group (Fig. S7-A). Importantly, there was no difference in mean amylase or lipase levels (markers of acute pancreatitis) (27) in the serum samples between treatment and control groups (Fig. S7-B), and no overt effects on comprehensive metabolic panel markers (Fig. S7-C \& D) or weekly body weight (Fig S8) was observed.

\section{DISCUSSION}

Previous studies demonstrated that SPINK1 outlier expression identified a subset of ETS negative prostate cancers ( $10 \%$ of all PSA-screened prostate cancers), although the mechanism for SPINK1 outlier expression remains unknown (10). SPINK1 defines a distinct molecular sub-type of prostate cancer characterized by lack of ETS gene fusions as well as a more aggressive phenotype as corroborated by independent groups across distinct cohorts of prostate cancer patients $(10,21)$. Thus, our working hypothesis is that $S P I N K 1^{+}$prostate cancer represents an aggressive form of prostate cancer that may respond to different therapies than ETS gene fusion positive prostate cancers.

Here, we show that SPINK1 promotes prostate cancer proliferation and invasion through autocrine and paracrine signaling. We also demonstrate an in vivo role for SPINK1 in intravasation and tumor xenograft growth. At present, the precise mechanism and signaling pathways responsible for these effects in $S P I N K 1^{+}$prostate cancer is unclear. A recent study showed that mutation of SPINK1 at leucine 18 (L18) in the trypsin interaction site reduced tumor growth, angiogenesis, and lung metastases in HT-29 5M21 human colon carcinoma tumor xenografts, suggesting that the cancer-related phenotypes of SPINK1 may be related to its anti-proteinase activity (28). Moreover, the invasive behavior of these HT-29 5M21 colon cancer cells was abolished using anti-SPINK1 antibody (28). However in our study, we did not observe any effect of SPINK1 on trypsin or PSA, two candidate proteases in prostate cancer.

Recent studies also indicate that SPINK1 may be an apoptosis inhibitor preventing serine protease-dependent cell death (29). In this study, we show that SPINK1, which has structural similarities with EGF (30), binds to EGFR, and inhibiting SPINK1 attenuates key downstream mediators of the EGFR pathway including MEK, ERK and AKT. Furthermore, we also show that SPINK1 dimerizes EGFR and induces sustained phosphorylation of EGFR, which have been shown to be critical for downstream signaling activation after ligand binding (31). However, in contrast to SPINK1 mAb, EGFR mAb only partially inhibited the cell invasive effects of 22RV1 cells and had no effect on cell proliferation, suggesting that SPINK1 engages both EGFR dependent and independent pathways to mediate its oncogenic effects. SPINK1 has also been shown to engage the EGFR/ mitogenactivated protein kinase cascade in NIH3T3 fibroblasts and pancreatic cancer cells (32). 
Taken together, this study provides compelling evidence that SPINK1 over-expression is oncogenic in prostate cancer and that inhibition of SPINKI via RNA interference or blocking antibodies may have therapeutic potential. Our pre-clinical models suggest that this therapeutic effect would only be effective in patients with $S P I N K 1^{+}$prostate cancer, suggesting that such therapies would need to be evaluated in a molecularly guided fashion. Although we did not formally test for long-term toxicity, we observed no morbidity or weight loss in mice treated with an anti-SPINK1 mAb. Furthermore, while the pancreas expresses high levels of SPINK1, we observed no evidence of pancreatitis by anti-SPINK1 $\mathrm{mAb}$ treatment, as evidenced by no significant change in serum lipase and amylase levels or other metabolic biomarkers.

As the area of antibody-based therapeutics for extracellular targets is well developed, based on examples such as trastuzumab in breast cancers with ERBB2 over-expression, we postulate that a SPINK1 blocking antibody may have similar efficacy on a molecularly defined subset of prostate cancers. We have previously demonstrated that patients with the subset of $S P I N K 1^{+} /$ETS $^{-}$prostate cancers can be reliably identified by immunohistochemistry $(10,20)$, as would be required for a molecularly defined clinical trial. While humanized SPINK1 mAbs are not yet available for clinical testing, our studies show that SPINK1 partially mediates its oncogenic effects through EGFR.

This finding prompted us to evaluate the utility of the FDA-approved EGFR mAb, cetuximab, which showed in vitro and in vivo activity only against $S P I N K 1^{+}$prostate cancer cells (although less effective than SPINK1 mAb). Phase I/II clinical trials of cetuximab (33) and EGFR small molecules have been largely disappointing in metastatic prostate cancer $(34,35)$; however, a small subset of patients have had responses, including 3 of 36 (8\%) patients who showed $>50 \%$ PSA decline in a phase Ib/IIa clinical trial of cetuximab in combination with doxorubicin in castrate- resistant metastatic prostate cancer patients (33). Results from our study provide a plausible mechanism for why only the limited subset of patients with positive cancers ( $\sim 10 \%$ of all cases) may benefit from EGFR inhibition. This hypothesis can be assessed retrospectively and in biomarker-informed clinical trials of patients with SPINKI ${ }^{+}$prostate cancer. Our results credential SPINKI as an oncogene in a subset of prostate cancers that can be molecularly identified and provides the rationale to develop humanized anti-SPINK1 antibodies for human clinical trials. Importantly, our work also supports the molecular sub-classification of prostate cancer in clinical trials (whether through SPINK/ETS status or other relevant biomarkers), which has lagged behind other common epithelial cancers (i.e., breast, lung, colon).

\section{MATERIALS AND METHODS}

\section{Cell lines and SPINK1 Knockdown}

The benign immortalized prostate cell line RWPE, prostate cancer cell lines DU145, PC3 and 22RV1 were obtained from the American Type Culture Collection (ATCC) and were grown according to ATCC guidelines. For stable knockdown of SPINK1, human lentiviral shRNAmir individual clone (ID V2LHS_153419) targeting against SPINK1 or non-silencing lentiviral shRNAmir in GIPZ vectors were purchased from Open Biosystems (Thermo Scientific Open Biosystems). Details are available in Supplementary Material Experimental Procedures.

\section{Quantitative PCR (QPCR)}

Total RNA was isolated using miRNeasy mini kit following manufacturer's instruction (Qiagen). Complimentary DNA was synthesized from one microgram of total RNA, using SuperScript III (Invitrogen) in the presence of random primers. Quantitative PCR (qPCR) 
was performed using the StepOne Real Time PCR system (Applied Biosystems). Details and primers information are available in Supplementary Material Experimental Procedures.

\section{Cell proliferation assay}

Proliferation for control and experimental cells was measured by a colorimetric assay based on the cleavage of the tetrazolium salt WST-1 by mitochondrial dehydrogenases (cell proliferation reagent WST1; Roche Diagnostics) at the indicated time points in triplicate. Cell counts for shNS vector and shSPINK1 cells were estimated by trypsinizing cells and analysis by Coulter counter (Beckman Coulter) at different time points in triplicates.

\section{Basement Membrane Matrix Invasion assay}

For invasion assays, shNS vector or shSPINK1 cells, RWPE, PC3 and 22RV1 cells were used. Equal numbers of the indicated cells were seeded onto the basement membrane matrix (BD Biosciences) present in the insert of a 24-well culture plate, RPMI media supplemented with $10 \%$ fetal bovine serum added to the lower chamber as a chemoattractant. After $48 \mathrm{hr}$, non-invading cells and EC matrix were removed using a cotton swab. Invaded cells were stained with crystal violet and photographed. The inserts were treated with $10 \%$ acetic acid, and absorbance was measured at $560 \mathrm{~nm}$.

\section{Chick Chorioallantoic Membrane (CAM) Assay}

The assay was performed essentially as described (26). Two million RWPE cells were mixed with either 200ng multiple tag control protein or 200ng of rSPINK1 protein and applied to the chorioallantoic membrane (CAM) of 11-day old chicken embryo. Similarly, two million 22RV1 or PC3 cells were mixed with either monoclonal IgG or anti-SPINK1 or C225 $(1 \mu \mathrm{g} / \mathrm{ml})$ and applied onto the upper CAM of a fertilized chicken embryo. Three days post-implantation, the relative number of cells that intravasate into the vasculature of the lower CAM was analyzed by extracting genomic DNA using the Purgene DNA purification system. Quantification of the human cells in the extracted DNA was done as described (36).

\section{RV1 and PC3 Xenograft Models}

Four week old male Balb/C nu/nu mice were purchased from Charles River, Inc. (Charles River Laboratory). Stable 22RV1 shNS-luciferase and 22RV1 shSPINKI-luciferase cells (5 $\times 10^{5}$ cells $)$, or $22 \mathrm{RV} 1$-Luc $\left(2 \times 10^{5}\right.$ cells $)$ or PC 3 -Luc $\left(5 \times 10^{5}\right.$ cells $)$ were resuspended in $100 \mu \mathrm{l}$ of saline with $20 \%$ Matrigel (BD Biosciences) and were implanted subcutaneously into the left flank regions of the mice. Details are available in Supplementary Material Experimental Procedures.

\section{Statistical analysis}

All values presented in the study were expressed as mean $+/$ - SEM. The significant differences between the groups were analyzed by a Student's $t$ test and a $P$ value of $<0.05$ or $<0.001$ were considered significant.

\section{Supplementary Material}

Refer to Web version on PubMed Central for supplementary material.

\section{Acknowledgments}

We thank Xia Jiang, Xiaojun Jing, Xiaoju Wang, Anastasia Yocum, Javed Siddiqui, Khalid Suleman, Rohit Mehra and Christopher A. Maher for the technical assistance, Mohan Dhanasekaran and Chad Brenner for discussions, and Jill Granger for critically reading the manuscript. This work is supported in part by the Department of Defense W81XWH-08-1-0031, Early Detection Research Network UO1 CA111275, Prostate SPORE P50CA69568, and 
National Institutes of Health (R01CA132874). A.M.C. is supported by the Doris Duke Charitable Foundation Clinical Scientist Award, Burroughs Welcome Foundation Award in Clinical Translational Research and the Prostate Cancer Foundation (PCF). A.M.C. is an American Cancer Society Research Professor. B.A is supported by the Genentech Foundation Postdoctoral Fellowship and Young Investigator Award from the Expedition Inspiration Fund for Breast Cancer Research. S.A.T. is supported by a Young Investigator Award from the PCF. Q.C. is supported by U.S. Department of Defense (PC094725). S.V. is supported by Prostate Cancer SPORE Career Development award.

\section{REFERENCES AND NOTES}

1. Jemal A, Siegel R, Xu J, Ward E. Cancer statistics, 2010. CA Cancer J Clin. 2010; 60:277-300. [PubMed: 20610543]

2. Helgeson BE, Tomlins SA, Shah N, Laxman B, Cao Q, Prensner JR, Cao X, Singla N, Montie JE, Varambally S, Mehra R, Chinnaiyan AM. Characterization of TMPRSS2:ETV5 and SLC45A3:ETV5 gene fusions in prostate cancer. Cancer Res. 2008; 68:73-80. [PubMed: 18172298]

3. Tomlins SA, Rhodes DR, Perner S, Dhanasekaran SM, Mehra R, Sun XW, Varambally S, Cao X, Tchinda J, Kuefer R, Lee C, Montie JE, Shah RB, Pienta KJ, Rubin MA, Chinnaiyan AM. Recurrent fusion of TMPRSS2 and ETS transcription factor genes in prostate cancer. Science. 2005; 310:644-648. [PubMed: 16254181]

4. Tomlins SA, Mehra R, Rhodes DR, Smith LR, Roulston D, Helgeson BE, Cao X, Wei JT, Rubin MA, Shah RB, Chinnaiyan AM. TMPRSS2:ETV4 gene fusions define a third molecular subtype of prostate cancer. Cancer Res. 2006; 66:3396-3400. [PubMed: 16585160]

5. Tomlins SA, Laxman B, Dhanasekaran SM, Helgeson BE, Cao X, Morris DS, Menon A, Jing X, Cao Q, Han B, Yu J, Wang L, Montie JE, Rubin MA, Pienta KJ, Roulston D, Shah RB, Varambally S, Mehra R, Chinnaiyan AM. Distinct classes of chromosomal rearrangements create oncogenic ETS gene fusions in prostate cancer. Nature. 2007; 448:595-599. [PubMed: 17671502]

6. Tomlins SA, Bjartell A, Chinnaiyan AM, Jenster G, Nam RK, Rubin MA, Schalken JA. ETS Gene Fusions in Prostate Cancer: From Discovery to Daily Clinical Practice. Eur Urol. 2009; 56:275-286. [PubMed: 19409690]

7. Zong Y, Xin L, Goldstein AS, Lawson DA, Teitell MA, Witte ON. ETS family transcription factors collaborate with alternative signaling pathways to induce carcinoma from adult murine prostate cells. Proc Natl Acad Sci U S A. 2009

8. King JC, Xu J, Wongvipat J, Hieronymus H, Carver BS, Leung DH, Taylor BS, Sander C, Cardiff RD, Couto SS, Gerald WL, Sawyers CL. Cooperativity of TMPRSS2-ERG with PI3-kinase pathway activation in prostate oncogenesis. Nat Genet. 2009; 41:524-526. [PubMed: 19396167]

9. Carver BS, Tran J, Gopalan A, Chen Z, Shaikh S, Carracedo A, Alimonti A, Nardella C, Varmeh S, Scardino PT, Cordon-Cardo C, Gerald W, Pandolfi PP. Aberrant ERG expression cooperates with loss of PTEN to promote cancer progression in the prostate. Nat Genet. 2009; 41:619-624. [PubMed: 19396168]

10. Tomlins SA, Rhodes DR, Yu J, Varambally S, Mehra R, Perner S, Demichelis F, Helgeson BE, Laxman B, Morris DS, Cao Q, Cao X, Andren O, Fall K, Johnson L, Wei JT, Shah RB, AlAhmadie H, Eastham JA, Eggener SE, Fine SW, Hotakainen K, Stenman UH, Tsodikov A, Gerald WL, Lilja H, Reuter VE, Kantoff PW, Scardino PT, Rubin MA, Bjartell AS, Chinnaiyan AM. The role of SPINK1 in ETS rearrangement-negative prostate cancers. Cancer Cell. 2008; 13:519-528. [PubMed: 18538735]

11. Kazal LA, Spicer DS, Brahinsky RA. Isolation of a crystalline trypsin inhibitor-anticoagulant protein from pancreas. J Am Chem Soc. 1948; 70:3034-3040. [PubMed: 18882536]

12. Kelloniemi E, Rintala E, Finne P, Stenman UH. Tumor-associated trypsin inhibitor as a prognostic factor during follow-up of bladder cancer. Urology. 2003; 62:249-253. [PubMed: 12893328]

13. Lukkonen A, Lintula S, von Boguslawski K, Carpen O, Ljungberg B, Landberg G, Stenman UH. Tumor-associated trypsin inhibitor in normal and malignant renal tissue and in serum of renal-cell carcinoma patients. Int J Cancer. 1999; 83:486-490. [PubMed: 10508484]

14. Haglund C, Huhtala ML, Halila H, Nordling S, Roberts PJ, Scheinin TM, Stenman UH. Tumourassociated trypsin inhibitor, TATI, in patients with pancreatic cancer, pancreatitis and benign biliary diseases. Br J Cancer. 1986; 54:297-303. [PubMed: 3741764] 
15. Higashiyama M, Monden T, Tomita N, Murotani M, Kawasaki Y, Morimoto H, Murata A, Shimano T, Ogawa M, Mori T. Expression of pancreatic secretory trypsin inhibitor (PSTI) in colorectal cancer. Br J Cancer. 1990; 62:954-958. [PubMed: 2257226]

16. Huhtala ML, Kahanpaa K, Seppala M, Halila H, Stenman UH. Excretion of a tumor-associated trypsin inhibitor (TATI) in urine of patients with gynecological malignancy. Int J Cancer. 1983; 31:711-714. [PubMed: 6190763]

17. Paju A, Vartiainen J, Haglund C, Itkonen O, von Boguslawski K, Leminen A, Wahlstrom T, Stenman UH. Expression of trypsinogen-1, trypsinogen-2, and tumor-associated trypsin inhibitor in ovarian cancer: prognostic study on tissue and serum. Clin Cancer Res. 2004; 10:4761-4768. [PubMed: 15269150]

18. Ohmachi Y, Murata A, Matsuura N, Yasuda T, Monden M, Mori T, Ogawa M, Matsubara K. Specific expression of the pancreatic-secretory-trypsin-inhibitor (PSTI) gene in hepatocellular carcinoma. Int J Cancer. 1993; 55:728-734. [PubMed: 8244568]

19. Bjartell A, Paju A, Zhang WM, Gadaleanu V, Hansson J, Landberg G, Stenman UH. Expression of tumor-associated trypsinogens (TAT-1 and TAT-2) in prostate cancer. Prostate. 2005; 64:29-39. [PubMed: 15651064]

20. Laxman B, Morris DS, Yu J, Siddiqui J, Cao J, Mehra R, Lonigro RJ, Tsodikov A, Wei JT, Tomlins SA, Chinnaiyan AM. A first-generation multiplex biomarker analysis of urine for the early detection of prostate cancer. Cancer Res. 2008; 68:645-649. [PubMed: 18245462]

21. Leinonen KA, Tolonen TT, Bracken H, Stenman UH, Tammela TL, Saramaki OR, Visakorpi T. Association of SPINK1 expression and TMPRSS2:ERG fusion with prognosis in endocrinetreated prostate cancer. Clin Cancer Res. 16:2845-2851. [PubMed: 20442300]

22. Hotakainen K, Bjartell A, Sankila A, Jarvinen R, Paju A, Rintala E, Haglund C, Stenman UH. Differential expression of trypsinogen and tumor-associated trypsin inhibitor (TATI) in bladder cancer. Int J Oncol. 2006; 28:95-101. [PubMed: 16327984]

23. Dagvadorj A, Tan SH, Liao Z, Cavalli LR, Haddad BR, Nevalainen MT. Androgen-regulated and highly tumorigenic human prostate cancer cell line established from a transplantable primary CWR22 tumor. Clin Cancer Res. 2008; 14:6062-6072. [PubMed: 18829484]

24. Hunt LT, Barker WC, Dayhoff MO. Epidermal growth factor: internal duplication and probable relationship to pancreatic secretory trypsin inhibitor. Biochem Biophys Res Commun. 1974; 60:1020-1028. [PubMed: 4429557]

25. Bartelt DC, Shapanka R, Greene LJ. The primary structure of the human pancreatic secretory trypsin inhibitor. Amino acid sequence of the reduced S-aminoethylated protein. Arch Biochem Biophys. 1977; 179:189-199. [PubMed: 843082]

26. Zijlstra A, Mellor R, Panzarella G, Aimes RT, Hooper JD, Marchenko ND, Quigley JP. A quantitative analysis of rate-limiting steps in the metastatic cascade using human-specific real-time polymerase chain reaction. Cancer Res. 2002; 62:7083-7092. [PubMed: 12460930]

27. Jung WS, Chae YS, Kim DY, Seo SW, Park HJ, Bae GS, Kim TH, Oh HJ, Yun KJ, Park RK, Kim JS, Kim EC, Hwang SY, Park SJ, Song HJ. Gardenia jasminoides protects against ceruleininduced acute pancreatitis. World J Gastroenterol. 2008; 14:6188-6194. [PubMed: 18985809]

28. Gouyer V, Fontaine D, Dumont P, de Wever O, Fontayne-Devaud H, Leteurtre E, Truant S, Delacour D, Drobecq H, Kerckaert JP, de Launoit Y, Bracke M, Gespach C, Desseyn JL, Huet G. Autocrine induction of invasion and metastasis by tumor-associated trypsin inhibitor in human colon cancer cells. Oncogene. 2008; 27:4024-4033. [PubMed: 18317448]

29. Lu X, Lamontagne J, Lu F, Block TM. Tumor-associated protein SPIK/TATI suppresses serine protease dependent cell apoptosis. Apoptosis. 2008; 13:483-494. [PubMed: 18347987]

30. Scheving LA. Primary amino acid sequence similarity between human epidermal growth factorurogastrone, human pancreatic secretory trypsin inhibitor, and members of porcine secretin family. Arch Biochem Biophys. 1983; 226:411-413. [PubMed: 6605724]

31. Mendelsohn J, Baselga J. The EGF receptor family as targets for cancer therapy. Oncogene. 2000; 19:6550-6565. [PubMed: 11426640]

32. Ozaki N, Ohmuraya M, Hirota M, Ida S, Wang J, Takamori H, Higashiyama S, Baba H, Yamamura K. Serine protease inhibitor Kazal type 1 promotes proliferation of pancreatic cancer 
cells through the epidermal growth factor receptor. Mol Cancer Res. 2009; 7:1572-1581. [PubMed: 19737965]

33. Slovin SF, Kelly WK, Wilton A, Kattan M, Myskowski P, Mendelsohn J, Scher HI. Antiepidermal growth factor receptor monoclonal antibody cetuximab plus Doxorubicin in the treatment of metastatic castration-resistant prostate cancer. Clin Genitourin Cancer. 2009; 7:E7782. [PubMed: 19815486]

34. Nabhan C, Lestingi TM, Galvez A, Tolzien K, Kelby SK, Tsarwhas D, Newman S, Bitran JD. Erlotinib has moderate single-agent activity in chemotherapy-naive castration-resistant prostate cancer: final results of a phase II trial. Urology. 2009; 74:665-671. [PubMed: 19616281]

35. Pezaro C, Rosenthal MA, Gurney H, Davis ID, Underhill C, Boyer MJ, Kotasek D, Solomon B, Toner GC. An open-label, single-arm phase two trial of gefitinib in patients with advanced or metastatic castration-resistant prostate cancer. Am J Clin Oncol. 2009; 32:338-341. [PubMed: 19363437]

36. van der Horst EH, Leupold JH, Schubbert R, Ullrich A, Allgayer H. TaqMan-based quantification of invasive cells in the chick embryo metastasis assay. Biotechniques. 2004; 37:940-942. 944, 946. [PubMed: 15597543] 
A

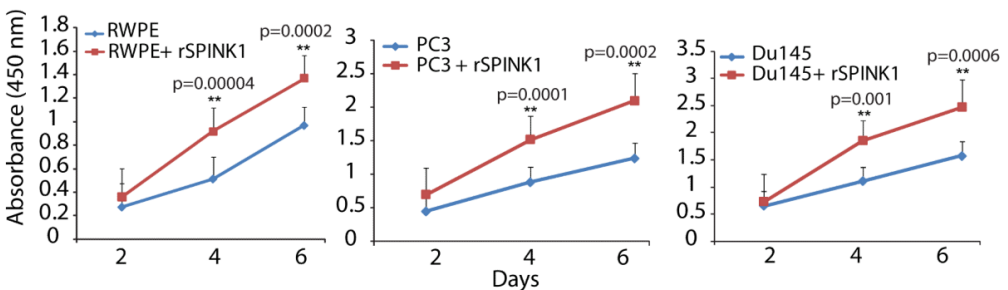

B

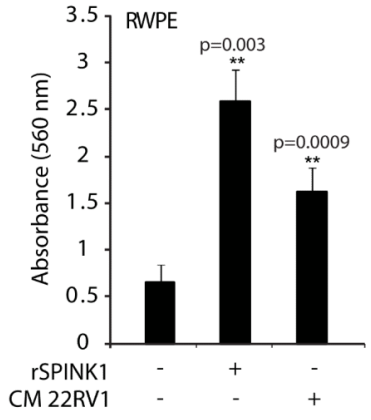

D
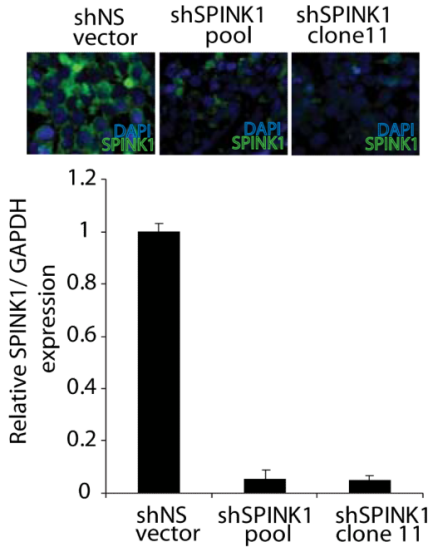

$\mathbf{F}$

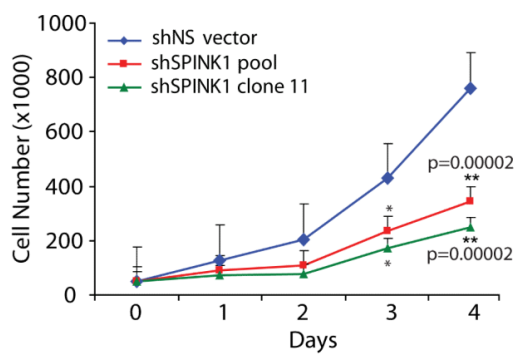

C

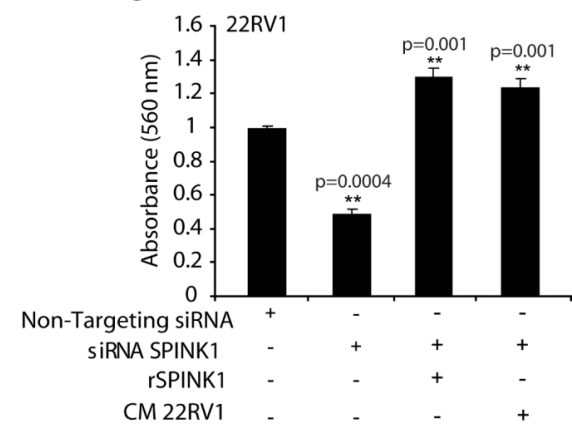

$\mathbf{E}$
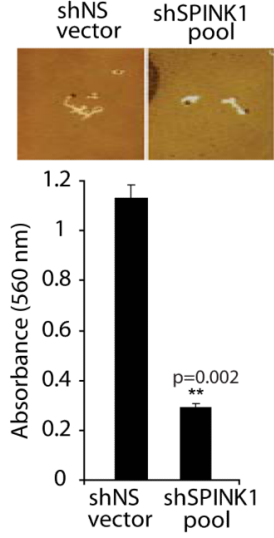

G

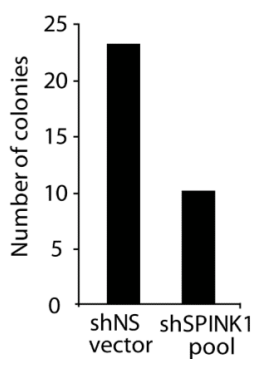

Figure 1.

Oncogenic in vitro effects of SPINK1 in prostate cells. (A) SPINK1 stimulates cell proliferation in SPINKI-/ETS ${ }^{-}$cell lines. Benign immortalized prostate cell line RWPE and prostate cancer cell lines DU145 and PC3 (all SPINK1 $/$ ETS $^{-}$) were untreated or treated with $10 \mathrm{ng} / \mathrm{ml}$ of rSPINK1. Cell proliferation was measured by a WST-1 colorimetric assay at the indicated time points. (B) SPINK1 mediates invasion of RWPE cells as measured by Boyden chamber Matrigel invasion assay. RWPE cells were treated with $10 \mathrm{ng} / \mathrm{ml}$ of rSPINK1 or conditioned media (CM) from 22RV1 cells $\left(S P I N K 1^{+} / E T S^{-}\right)$. (C) As in $\mathbf{B}$, except using 22RV1 cells transfected with siRNA against SPINK1. SPINK1 silenced 22RV1 cells were further treated with $10 \mathrm{ng} / \mathrm{ml}$ of rSPINK1 or CM from 22RV1 cells. (D) SPINK1 
expression in SPINK1 knockdown 22RV1 cells (stable pooled shSPINK1 or stable shSPINK1 clone 11) compared to non-targeting pooled stable control (shNS vector) cells by quantitative PCR (transcript) or immunofluorescence using an antibody against SPINK1 (protein, upper inset; 600X magnification). (E) Invasion assay using shSPINKI and $\operatorname{sh} N S$ cells. Representative photomicrographs (400X magnification) showing cell motility assay (top inset) are shown. sh $N S$ vector cells exhibit longer cell motility tracks as compared to shSPINK1 knockdown cells. (F) Cell proliferation assay using pooled shSPINK1, shSPINK1 clone 11, or shNS cells at the indicated time points. (G) Soft agar colony assay using pooled shSPINK1 cells and shNS cells. All experiments were independently performed in triplicate. Data shown represents mean +/- SEM. P values from significant two-sided Student's $t$ tests are given $(*=<0.05, * *=<0.001)$. 
A

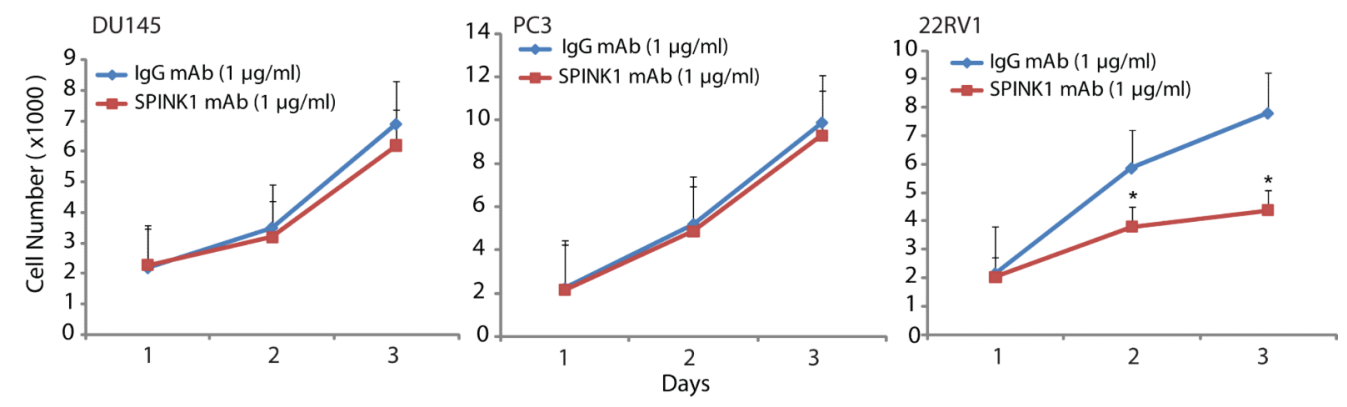

B

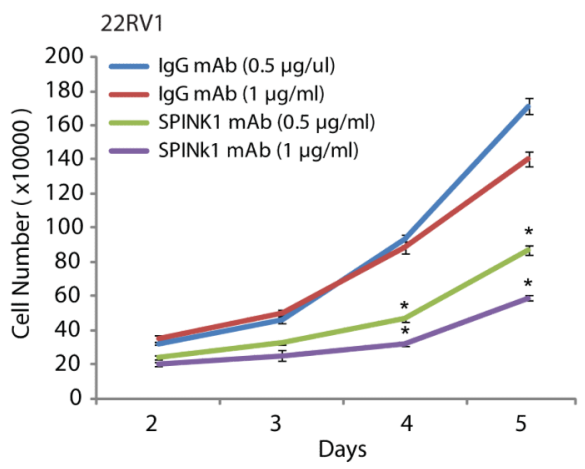

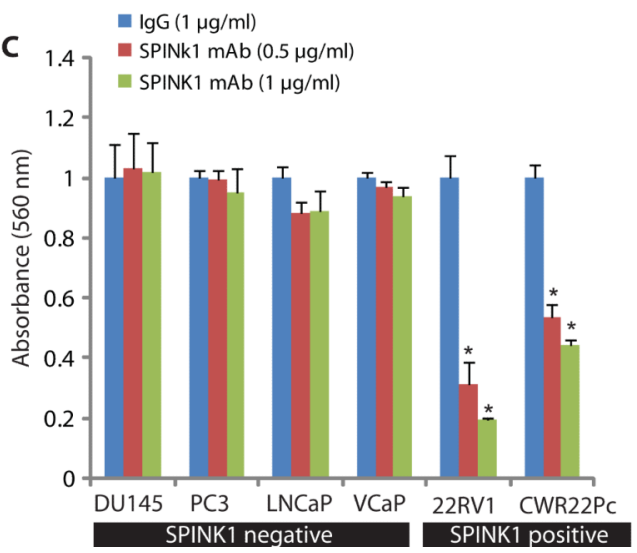

Figure 2.

Anti-SPINK1 mAb attenuates in vitro proliferation and invasion exclusively in $S P I N K 1^{+} /$ ETS ${ }^{-}$prostate cancer cells. (A) Cell proliferation of DU145, PC3 and 22RV1 cells was assessed in the presence of $1 \mu \mathrm{g} / \mathrm{ml}$ SPINK1 mAb or IgG mAb (B) As in A except using 22RV1 cells and $0.5-1 \mu \mathrm{g} / \mathrm{ml}$ SPINK1 mAb or IgG mAb. (C) Effect of SPINK1 mAb or IgG $\mathrm{mAb}$ on invasion of SPINK1+/ETS- cells (22RV1 and CWR22PC) and SPINK1 ${ }^{+} /$ETS $^{-}$cells (DU145, PC3, LNCaP and VCaP) All experiments were independently performed in triplicates. Data shown represents mean +/- SEM. P values from significant two-sided Student's t tests are given $(*=<0.05, * *=<0.001)$. 
A

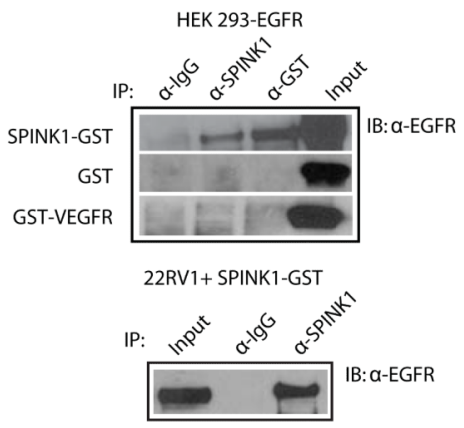

B

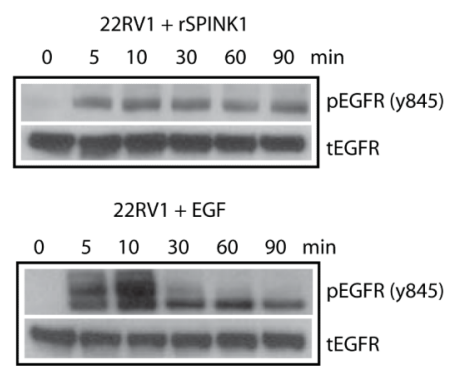

C

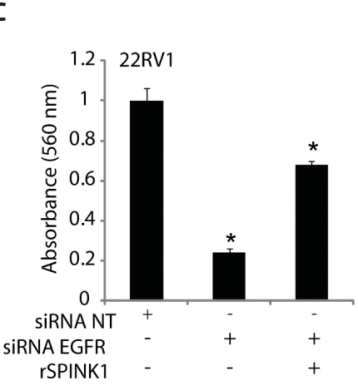

F

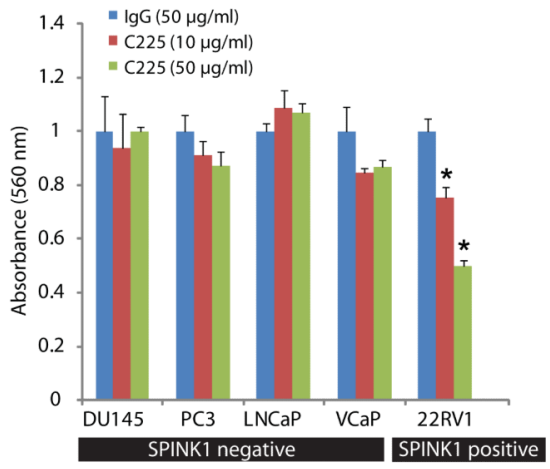

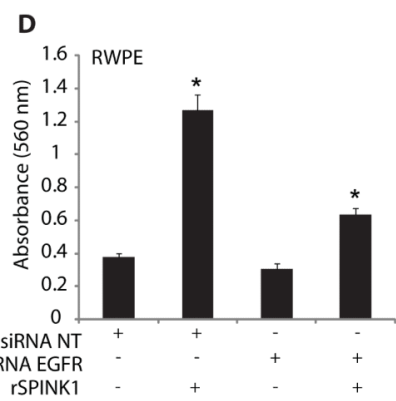

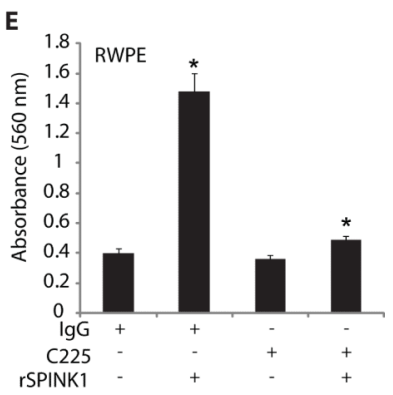

G

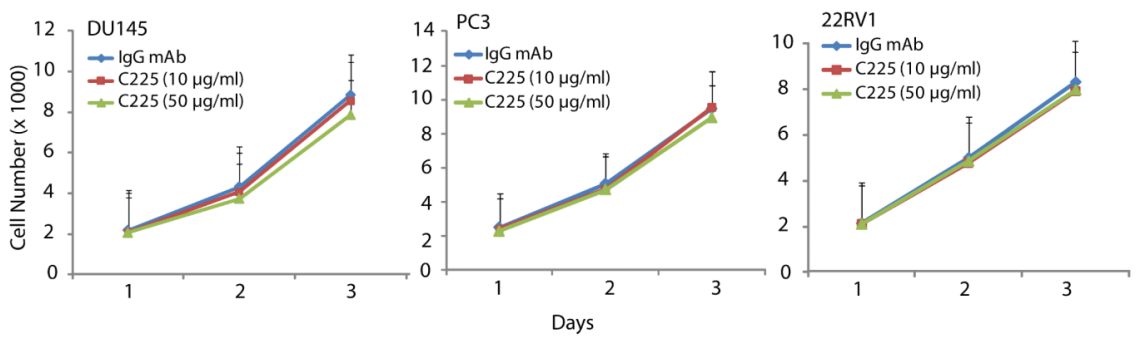

Figure 3.

SPINK1 mediates its oncogenic effects in part through EGFR (A) Immunoprecipitation using anti-IgG, anti-SPINK1 or anti-GST of exogenous SPINK1-GST, GST or GSTVEGFR added to HEK-293 cells transfected with EGFR and immunoblotted with antiEGFR (top panel), and immunoprecipitation using anti-IgG or anti-SPINK1 of exogenous SPINK1-GST added to 22RV1 cells and immunoblotted with anti-EGFR (bottom panel) (B) Western blot showing EGFR phosphorylation in response to rSPINK1 (100 ng/ml) or EGF (10 ng/ml) stimulation. (C) Invasion assay showing siRNA mediated EGFR knockdown 22RV1 cells treated with $10 \mathrm{ng} / \mathrm{ml}$ of rSPINK1 (D) Same as in C, except with RWPE cells. (E) Invasion assay showing rSPINK1 (10 ng/ml) stimulated RWPE cells in the 
presence or absence of C225 (cetuximab, $50 \mu \mathrm{g} / \mathrm{ml}$ ) or IgG mAb (50 $\mu \mathrm{g} / \mathrm{ml})(\mathbf{F})$ Invasion assay showing the effect of $\mathrm{IgG}$ or $\mathrm{C} 225$ antibody on $S P I N K 1^{+}$and $S P I N K 1^{-}$cancer cells. (G) As in $\mathbf{F}$, except $22 \mathrm{RV} 1$ cells were treated with a combination of anti-SPINK1 and/or $\mathrm{C} 225 \mathrm{mAb}(1 \mu \mathrm{g} / \mathrm{ml}$ and $50 \mu \mathrm{g} / \mathrm{ml}$ respectively). (H) Cell proliferation assay using the indicated cells in the presence of $\operatorname{IgG} \mathrm{mAb}$ or $\mathrm{C} 225$. All experiments were independently performed in triplicates. Data shown represents mean $+/$ - SEM. P values from significant two-sided Student's $\mathrm{t}$ tests are given $(*=<0.05, * *=<0.001)$. 

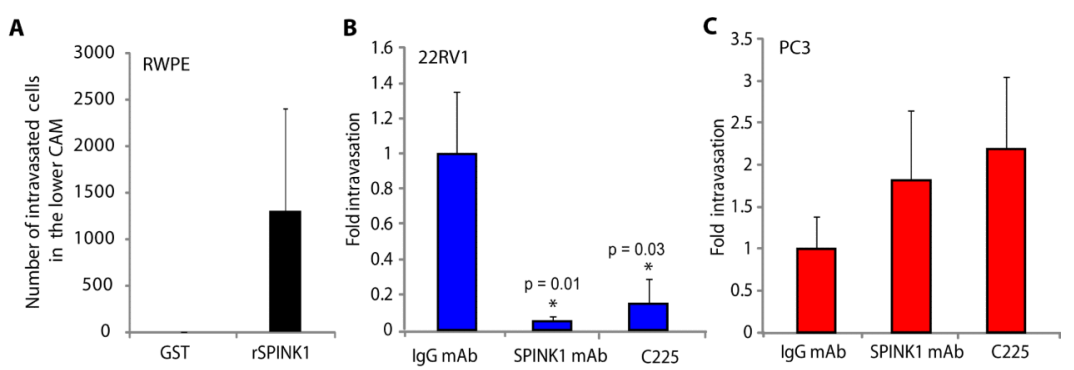
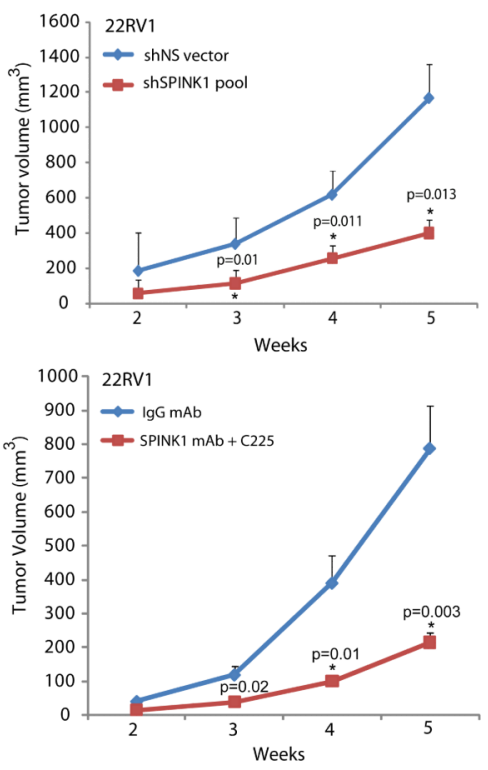

H
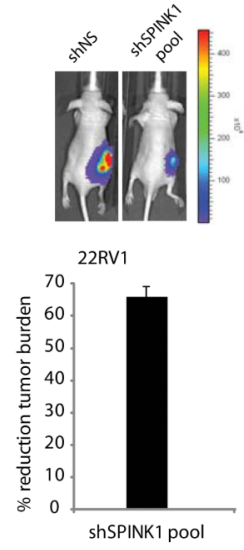

E
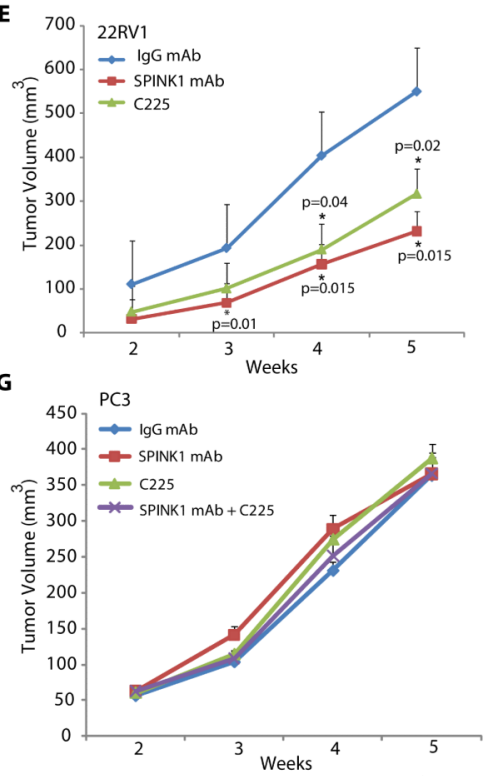

I

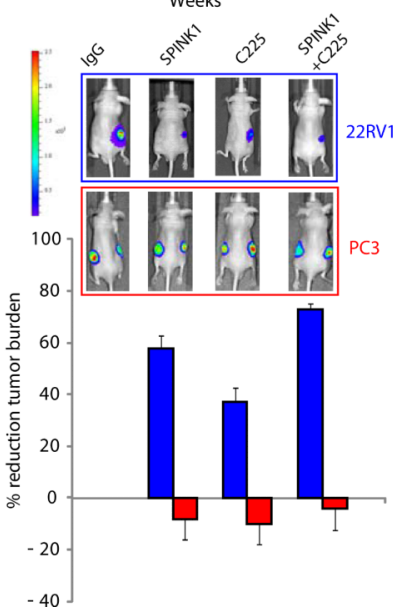

Figure 4.

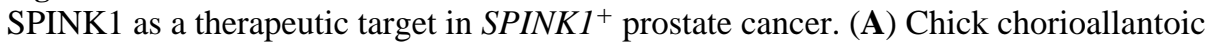
membrane (CAM) assay quantifying intravasated RWPE cells upon stimulation with rSPINK1 ( $\mathrm{n}=6$ in each group). (B) CAM assay using 22RV1 cells in the presence of $\operatorname{IgG}$ $\mathrm{mAb}$, SPINK1 $\mathrm{mAb}$ or $\mathrm{C} 225$ ( $\mathrm{n}=5$ in each group), with fold change of intravasated cells compared to IgG mAb plotted. (C) As in B, except using PC3 cells. (D) Subcutaneous xenograft growth of shNS-luciferase (luc) or shSPINK1-luc 22RV1 cells implanted in male $\mathrm{BALB} / \mathrm{C}$ nu/nu mice ( $\mathrm{n}=10$ in each group). (E) As in D, except using 22RV1-luc cell xenografts treated with control IgG mAb (n=8), SPINK1 mAb (n=6) or C225 (n=8) $(10 \mathrm{mg} /$ $\mathrm{kg}$ body weight) twice a week. (F) Same as in $\mathbf{E}$, except mice (n=7 per group) were treated 
with a combination of SPINK $1 \mathrm{mAb}$ and $\mathrm{C} 225 \mathrm{mAb}$ (10 mg/kg body weight for both). (G) As in $\mathbf{E} \boldsymbol{\&} \mathbf{F}$, except using PC3-luc xenografts treated with control IgG mAb, SPINK1 mAb or $\mathrm{C} 225$ ( $\mathrm{n}=8$ per group) $(10 \mathrm{mg} / \mathrm{kg}$ body weight) alone or in combination twice a week. (H) Representative bioluminescence images from mice in $\mathbf{D}$ bearing pooled shNS-luc or shSPINK1-luc xenografts and \% reduction in tumor volume at week 5. (I) Same as $\mathbf{H}$, except bioluminescence images from mice bearing 22RV1-luc xenografts in (red, top panel) or PC3-luc (blue, lower panel) mice treated with IgG mAb, SPINK1 mAb, or C225 mAb alone or in combination, with comparative \% reduction plot in tumor volume at week 5 . Data shown represents mean +/- SEM. P values from significant two-sided Student's $t$ tests are given $(*=<0.05, * *=<0.001)$. 